\title{
Force Field Parameter Development for the Thiolate/Defective Au (111) Interface
}

Guobing Zhou, ${ }^{\mathrm{a}}$ Chang Liu, ${ }^{\mathrm{b}}$ Lloyd A. Bumm, ${ }^{\mathrm{c}}$ Liangliang Huang ${ }^{* a}$

(a) School of Chemical, Biological and Materials Engineering, University of Oklahoma, Norman, Oklahoma 73019, United States

(b) State Key Laboratory of Materials-Oriented Chemical Engineering, Nanjing Tech University, Nanjing 210009, China

(c) Homer L. Dodge Department of Physics and Astronomy, University of Oklahoma, Norman, Oklahoma 73019, United States

\section{Supporting Information 2}

This document shows the steps to develop the force constants for bond, angle, and torsional terms studied in the present study. Files mentioned in the text below are provided for your reference and all executables mentioned below are for Windows OS, and the Linux version can be found on the website (https://dasher.wustl.edu/tinker).

Part 1: Procedures to obtain the force constants for bonds and angles via matching the vibrational frequency.

\section{Needed softwares:}

(1) Tinker-Ver. 7.1 (https://dasher.wustl.edu/tinker/)

(2) Force Field Explorer-Ver. 6.0 (https://dasher.wustl.edu/tinker/) 
(3) VASP-Ver. 5.4.1

(4) Jmol-Ver. 14.29.26 (http://jmol.sourceforge.net/)

(5) VESTA-Ver. 3.4.7 (http://jp-minerals.org/vesta/en/)

(6) Avogadro-Ver. 1.2.0 (https://avogadro.cc/)

\section{Steps:}

1. Structural optimization (see files in 1_optimization folder): this part of optimization is for the initial staple motif structure on the Au (111) surface.

2. Vibrational frequency calculation (see files in 2_vibrational frequency calculation folder); 3. Convert CONTCAR to xyz (all files mentioned below are in 3_contcar2xyz folder)

(1) Open "CONTCAR" in 2_vibrational frequency analysis folder with VESTA

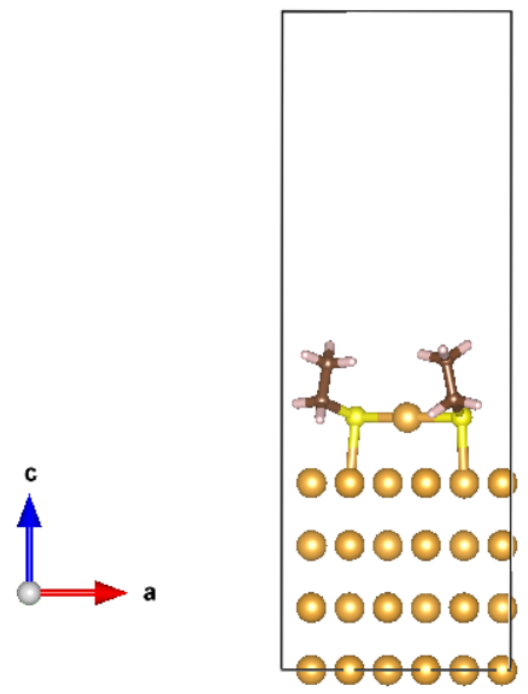

(2) From the "File" menu, choose "Export Data", save the file in pdb format in 3_contcar2xyz folder. By now, you shall have a file, named "staple.pdb".

(3) Open staple.pdb file with Avogadro (see the left figure below) and then delete all the bonds between the Au atoms in the substrate (see the right figure below) 

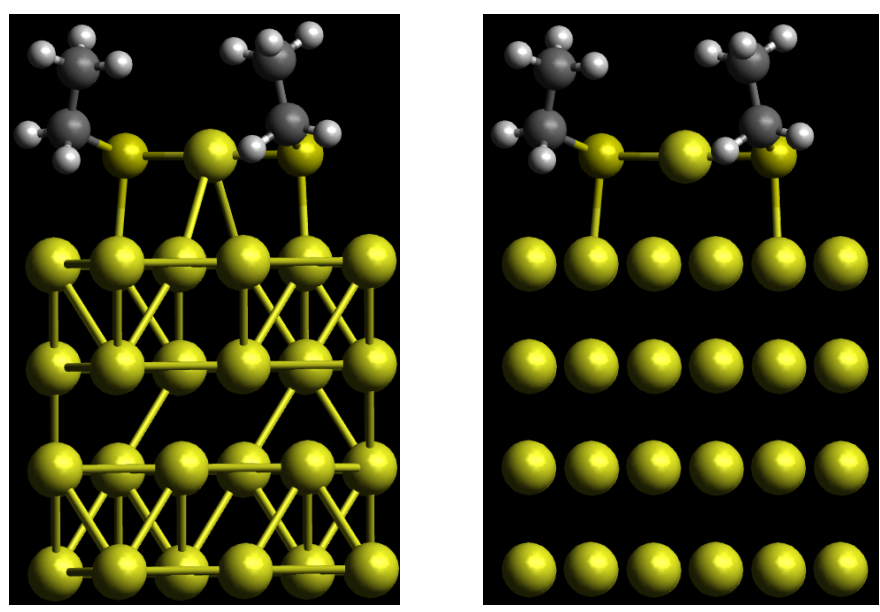

(4) From the "File" menu, choose "Save As", save the file in the mol2 format. By now, you shall have a file, named "staple.mol2"

(5) Convert the staple.mol2 file to .xyz file (Note: the xyz file of Tinker has a different format from the general xyz format).

(I) Double click the sybylxyz.exe executable and then import the staple.mol2 file. A new file named "staple.xyz" will be generated after pressing the enter button.
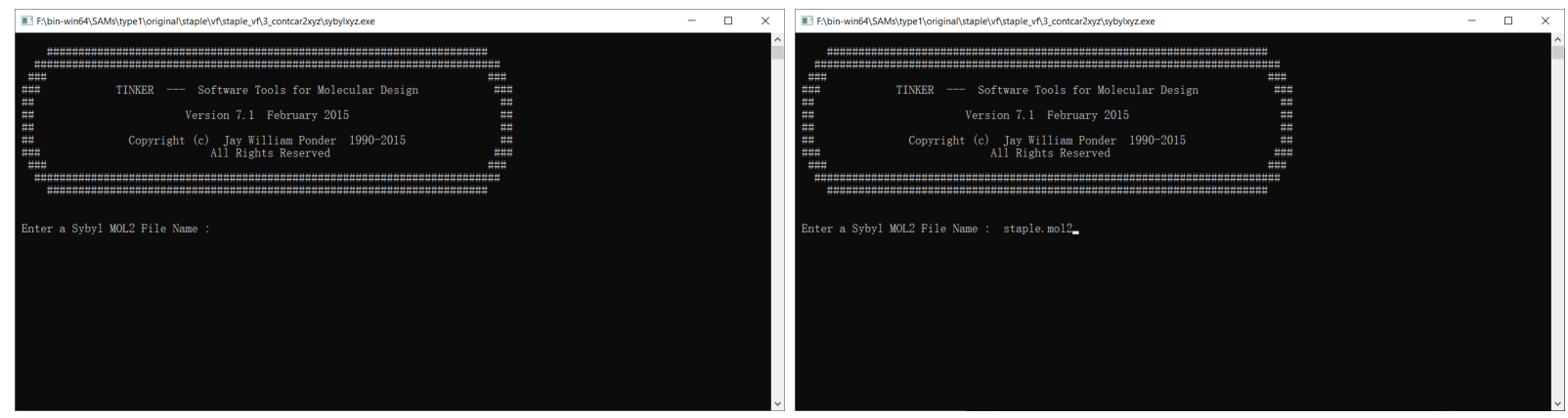

(II) Prepare the .key file, which contains simulation settings and parameters. Visit the website (https://dasher.wustl.edu/tinker/) for more details about the .key file. The descriptions of the .key file are listed below. To successfully run the following steps, you need to assign initial guess values for the force constants of the bonds, angles, and dihedrals (Note: 0.0 is not always a good choice since it may result in some errors). 


$\begin{array}{lrrr}\text { Atom } & \text { Atomic } & \text { Atomic } & \text { Bond } \\ \text { Type } & \text { Comment } & \text { Number } & \text { Weight } \\ & & \text { Number }\end{array}$

$\begin{array}{llllrrr}\text { atom } & 1 & \text { Au } & \text { "bulk Au atom" } & 79 & 196.966 & 0 \\ \text { atom } & 2 & \text { Au } & \text { "bulk Au atom bonded with S" } & 79 & 196.966 & 1 \\ \text { atom } & 3 & \text { Au } & \text { "Au adatom bonded with S" } & 79 & 196.966 & 2 \\ \text { atom } & 4 & \text { S } & \text { "S atom in C2S thiolate" } & 16 & 32.065 & 3 \\ \text { atom } & 5 & \text { C } & \text { "C atom in CH2" } & 6 & 12.027 & 4 \\ \text { atom } & 6 & \text { C } & \text { "C atom in CH3" } & 6 & 12.027 & 4 \\ \text { atom } & 7 & \text { H } & \text { "H atom in CH2 and CH3" } & 1 & 1.008 & 1\end{array}$

(III) After getting the .key file (see the staple.key file in 3 contcar2xyz folder), open the staple.xyz file and add the atom type for each atom. To distinguish the edited xyz file and the original one, the original xyz file from step (I) was named as staple_2.xyz.

Atom Original staple.xyz (staple_2.xyz for now)

new staple.xyz Type

\begin{tabular}{|c|c|c|c|c|c|c|c|c|c|c|c|}
\hline 65 & \multicolumn{5}{|c|}{$x * * x *$} & \multicolumn{5}{|l|}{65} & \\
\hline 1 & $\mathrm{Au}$ & 1.127000 & 3.019000 & 0.002000 & 0 & 1 & $\mathrm{Au}$ & 1.127000 & 3.019000 & 0.002000 & 1 \\
\hline 2 & $\mathrm{Au}$ & 1.127000 & 8.041000 & 0.002000 & 0 & 2 & $\mathrm{Au}$ & 1.127000 & 8.041000 & 0.002000 & 1 \\
\hline 3 & $\mathrm{Au}$ & 2.577000 & 5.530000 & 0.002000 & 0 & 3 & $\mathrm{Au}$ & 2.577000 & 5.530000 & 0.002000 & 1 \\
\hline 4 & $\mathrm{Au}$ & 2.577000 & 0.509000 & 0.002000 & 0 & 4 & $\mathrm{Au}$ & 2.577000 & 0.509000 & 0.002000 & 1 \\
\hline 5 & Au & 4.026000 & 3.019000 & 0.002000 & 0 & 5 & $\mathrm{Au}$ & 4.026000 & 3.019000 & 0.002000 & 1 \\
\hline 6 & Au & 4.026000 & 8.041000 & 0.002000 & 0 & 6 & $\mathrm{Au}$ & 4.026000 & 8.041000 & 0.002000 & 1 \\
\hline 7 & $\mathrm{Au}$ & 5.476000 & 0.509000 & 0.002000 & 0 & 7 & $\mathrm{Au}$ & 5.476000 & 0.509000 & 0.002000 & 1 \\
\hline 8 & $\mathrm{Au}$ & 5.476000 & 5.530000 & 0.002000 & 0 & 8 & $\mathrm{Au}$ & 5.476000 & 5.530000 & 0.002000 & 1 \\
\hline 9 & $\mathrm{Au}$ & 6.925000 & 8.041000 & 0.002000 & 0 & 9 & $\mathrm{Au}$ & 6.925000 & 8.041000 & 0.002000 & 1 \\
\hline 10 & $\mathrm{Au}$ & 6.925000 & 3.019000 & 0.002000 & 0 & 10 & Au & 6.925000 & 3.019000 & 0.002000 & 1 \\
\hline 11 & $\mathrm{Au}$ & 8.375000 & 0.509000 & 0.002000 & 0 & 11 & $\mathrm{Au}$ & 8.375000 & 0.509000 & 0.002000 & 1 \\
\hline 12 & $\mathrm{Au}$ & 8.375000 & 5.530000 & 0.002000 & 0 & 12 & $\mathrm{Au}$ & 8.375000 & 5.530000 & 0.002000 & 1 \\
\hline 13 & $\mathrm{Au}$ & 1.127000 & 1.346000 & 2.369000 & 0 & 13 & $\mathrm{Au}$ & 1.127000 & 1.346000 & 2.369000 & 1 \\
\hline 14 & $\mathrm{Au}$ & 1.127000 & 6.367000 & 2.369000 & 0 & 14 & $\mathrm{Au}$ & 1.127000 & 6.367000 & 2.369000 & 1 \\
\hline 15 & $\mathrm{Au}$ & 2.577000 & 3.856000 & 2.369000 & 0 & 15 & $\mathrm{Au}$ & 2.577000 & 3.856000 & 2.369000 & 1 \\
\hline 16 & $\mathrm{Au}$ & 2.577000 & 8.878000 & 2.369000 & 0 & 16 & $\mathrm{Au}$ & 2.577000 & 8.878000 & 2.369000 & 1 \\
\hline 17 & $\mathrm{Au}$ & 4.026000 & 1.346000 & 2.369000 & 0 & 17 & Au & 4.026000 & 1.346000 & 2.369000 & 1 \\
\hline 18 & $\mathrm{Au}$ & 4.026000 & 6.367000 & 2.369000 & 0 & 18 & Au & 4.026000 & 6.367000 & 2.369000 & 1 \\
\hline 19 & $\mathrm{Au}$ & 5.476000 & 8.878000 & 2.369000 & 0 & 19 & $\mathrm{Au}$ & 5.476000 & 8.878000 & 2.369000 & 1 \\
\hline 20 & $\mathrm{Au}$ & 5.476000 & 3.856000 & 2.369000 & 0 & 20 & $\mathrm{Au}$ & 5.476000 & 3.856000 & 2.369000 & 1 \\
\hline 21 & $\mathrm{Au}$ & 6.925000 & 1.346000 & 2.369000 & 0 & 21 & Au & 6.925000 & 1.346000 & 2.369000 & 1 \\
\hline 22 & $\mathrm{Aus}$ & 6.925000 & 6.367000 & 2.369000 & 0 & 22 & $\mathrm{Au}$ & 6.925000 & 6.367000 & 2.369000 & 1 \\
\hline 23 & Au & 8.375000 & 3.856000 & 2.369000 & 0 & 23 & $\mathrm{Au}$ & 8.375000 & 3.856000 & 2.369000 & 1 \\
\hline 24 & $\mathrm{Au}$ & 8.375000 & 8.878000 & 2.369000 & 0 & 24 & $\mathrm{Au}$ & 8.375000 & 8.878000 & 2.369000 & 1 \\
\hline
\end{tabular}




\begin{tabular}{|c|c|c|c|c|c|c|c|c|c|}
\hline 40 & $\mathrm{Au}$ & 2.577000 & 5.530000 & 7.104000 & 0 & 51 & & & \\
\hline 41 & $\mathrm{Au}$ & 4.026000 & 3.0199000 & 7.1040000 & 0 & & & & \\
\hline 42 & $\mathrm{Au}$ & 4.026000 & 8.041000 & 7.104000 & 0 & & & & \\
\hline 43 & $\mathrm{Au}$ & 5.476000 & 0.5090000 & 7.104000 & 0 & & & & \\
\hline 44 & $\mathrm{Au}$ & 5.476000 & 5.5300000 & 7.104000 & 0 & & & & \\
\hline 45 & $\mathrm{Au}$ & 6.925000 & 8.041000 & 7.104000 & 0 & & & & \\
\hline 46 & Au & 6.925000 & 3.019000 & 7.104000 & 0 & 50 & & & \\
\hline 47 & $\mathrm{Au}$ & 8.375000 & 0.5090000 & 7.104000 & 0 & & & & \\
\hline 48 & $\mathrm{Au}$ & 8.375000 & 5.5300000 & 7.104000 & 0 & & & & \\
\hline 49 & $\mathrm{Au}$ & 4.760000 & 4.3000000 & 9.590000 & 0 & 50 & 51 & & \\
\hline 50 & $s$ & 6.821000 & 3.235000 & 9.605000 & 0 & 46 & 49 & 52 & \\
\hline 51 & s & 2.806000 & 5.546000 & 9.614000 & 0 & 40 & 49 & 54 & \\
\hline 52 & c & 6.518000 & 1.553000 & 10.279000 & 0 & 50 & 53 & 56 & 57 \\
\hline 53 & c & 6.227000 & 1.5900000 & 11.771000 & 0 & 52 & 58 & 59 & 60 \\
\hline 54 & c & 1.499000 & 4.404000 & 10.211000 & 0 & 51 & 55 & 61 & 62 \\
\hline 55 & c & 1.731000 & 3.923000 & 11.633000 & 0 & 54 & 63 & 64 & 65 \\
\hline 56 & н & 7.434000 & 0.989000 & 10.047000 & 0 & 52 & & & \\
\hline 57 & $\mathrm{H}$ & 5.697000 & 1.091000 & 9.710000 & 0 & 52 & & & \\
\hline 58 & H & 7.042000 & 2.071000 & 12.330000 & 0 & 53 & & & \\
\hline 59 & H & 6.109000 & 0.565000 & 12.153000 & 0 & 53 & & & \\
\hline 60 & H & 5.300000 & 2.144000 & 11.979000 & 0 & 53 & & & \\
\hline 61 & H & 1.435000 & 3.557000 & 9.507000 & 0 & 54 & & & \\
\hline 62 & H & 0.563000 & 4.973000 & 10.114000 & 0 & 54 & & & \\
\hline 63 & H & 2.649000 & 3.3200000 & 11.698000 & 0 & 55 & & & \\
\hline 64 & H & 0.883000 & 3.296000 & 11.948000 & 0 & 55 & & & \\
\hline 65 & H & 1.820000 & 4.761000 & 12.318000 & 0 & 55 & & & \\
\hline
\end{tabular}

\begin{tabular}{|c|c|c|c|c|c|c|c|c|c|}
\hline 40 & $\mathrm{Au}$ & 2.577000 & 5.530000 & 7.104000 & 2 & 51 & & & \\
\hline 41 & $\mathrm{Au}$ & 4.026000 & 3.019000 & 7.104000 & 1 & & & & \\
\hline 42 & Au & 4.026000 & 8.041000 & 7.104000 & 1 & & & & \\
\hline 43 & $\mathrm{Au}$ & 5.476000 & 0.509000 & 7.104000 & 1 & & & & \\
\hline 44 & Au & 5.476000 & 5.530000 & 7.104000 & 1 & & & & \\
\hline 45 & $\mathrm{Au}$ & 6.925000 & 8.041000 & 7.104000 & 1 & & & & \\
\hline 46 & Au & 6.925000 & 3.019000 & 7.104000 & 2 & 50 & & & \\
\hline 47 & $\mathrm{Au}$ & 8.375000 & 0.509000 & 7.104000 & 1 & & & & \\
\hline 48 & $\mathrm{Au}$ & 8.375000 & 5.530000 & 7.104000 & 1 & & & & \\
\hline 49 & Au & 4.760000 & 4.300000 & 9.590000 & 3 & 50 & 51 & & \\
\hline 50 & $\mathrm{~s}$ & 6.821000 & 3.235000 & 9.605000 & 4 & 46 & 49 & 52 & \\
\hline 51 & $s$ & 2.806000 & 5.546000 & 9.614000 & 4 & 40 & 49 & 54 & \\
\hline 52 & c & 6.518000 & 1.553000 & 10.279000 & 5 & 50 & 53 & 56 & 57 \\
\hline 53 & c & 6.227000 & 1.590000 & 11.771000 & 6 & 52 & 58 & 59 & 60 \\
\hline 54 & c & 1.499000 & 4.404000 & 10.211000 & 5 & 51 & 55 & 61 & 62 \\
\hline 55 & c & 1.731000 & 3.923000 & 11.633000 & 6 & 54 & 63 & 64 & 65 \\
\hline 56 & H & 7.434000 & 0.989000 & 10.047000 & 7 & 52 & & & \\
\hline 57 & H & 5.697000 & 1.091000 & 9.710000 & 7 & 52 & & & \\
\hline 58 & H & 7.042000 & 2.071000 & 12.330000 & 7 & 53 & & & \\
\hline 59 & H & 6.109000 & 0.565000 & 12.153000 & 7 & 53 & & & \\
\hline 60 & H & 5.300000 & 2.144000 & 11.979000 & 7 & 53 & & & \\
\hline 61 & H & 1.435000 & 3.557000 & 9.507000 & 7 & 54 & & & \\
\hline 62 & H & 0.563000 & 4.973000 & 10.114000 & 7 & 54 & & & \\
\hline 63 & H & 2.649000 & 3.320000 & 11.698000 & 7 & 55 & & & \\
\hline 64 & H & 0.883000 & 3.296000 & 11.948000 & 7 & 55 & & & \\
\hline 65 & $\mathrm{H}$ & 1.820000 & 4.761000 & 12.318000 & 7 & 55 & & & \\
\hline
\end{tabular}

(IV) Before moving forward, for the staple.xyz file, we need to translate the center of mass to the origin. Steps: (a) copy the staple.xyz and staple.key files to translate the center of mass to the origin folder; (b) double click the xyzedit.exe executable; (c) input the staple.xyz file and press the Enter button; (d) input the number of the desired choice (here we choose NO. 12) and press the Enter button. This operation will generate a new file named as staple.xyz_2. To distinguish these two files, the old stape.xyz was named as staple_2.xyz and the new staple.xyz_2 was named as staple.xyz. Then, copy staple.xyz and staple.key files to 4 _vibrational frequency fitting folder.
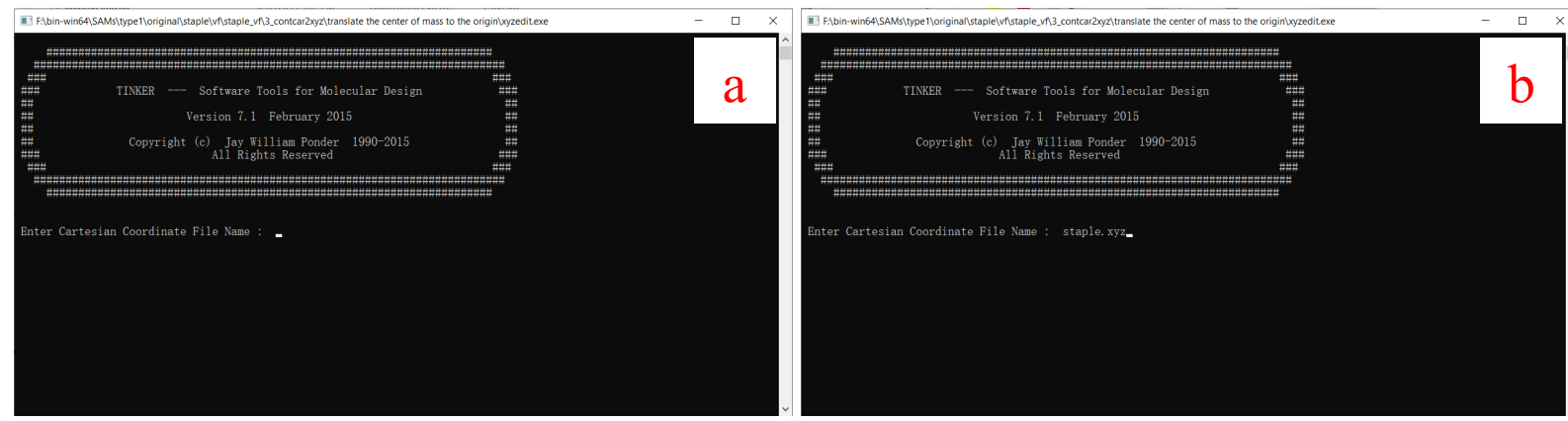


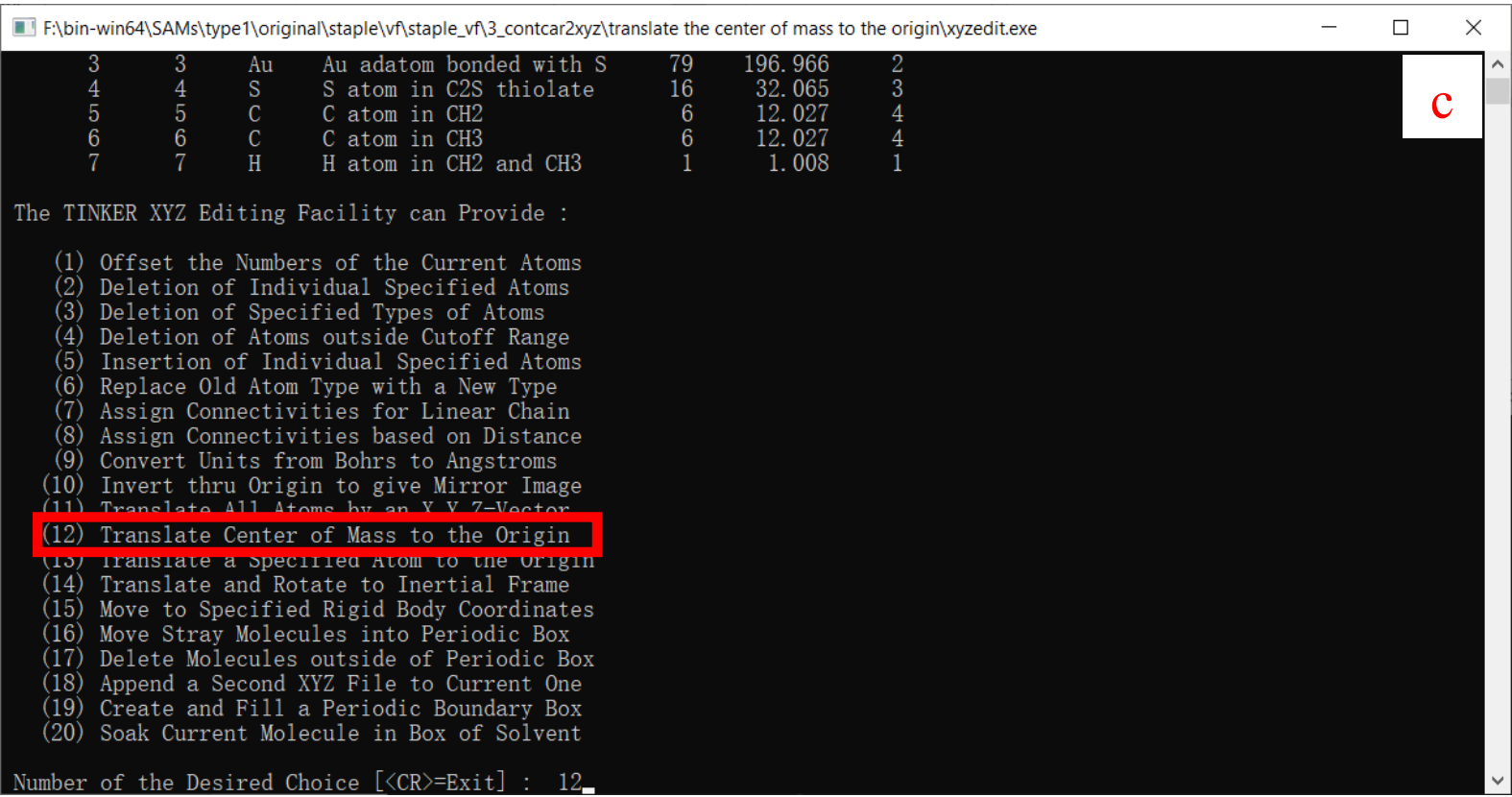

\section{Vibrational frequency fitting}

(1) Copy the OUTCAR file from the 2_vibrational frequency analysis folder. Open the OUTCAR file with Jmol to find the vibrational frequency for each vibrational mode.

(2) Click the "Tools" and select "AtomSetChooser".

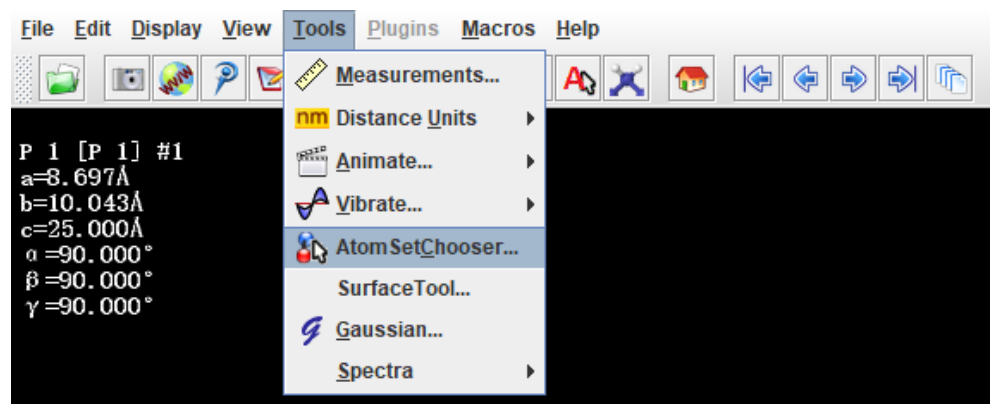

Scroll down to the end and then double click "Frequencies". Scroll down to find the vibrational spectra for the Au-S interface, which is in the range of $150-400 \mathrm{~cm}^{-1}$. 

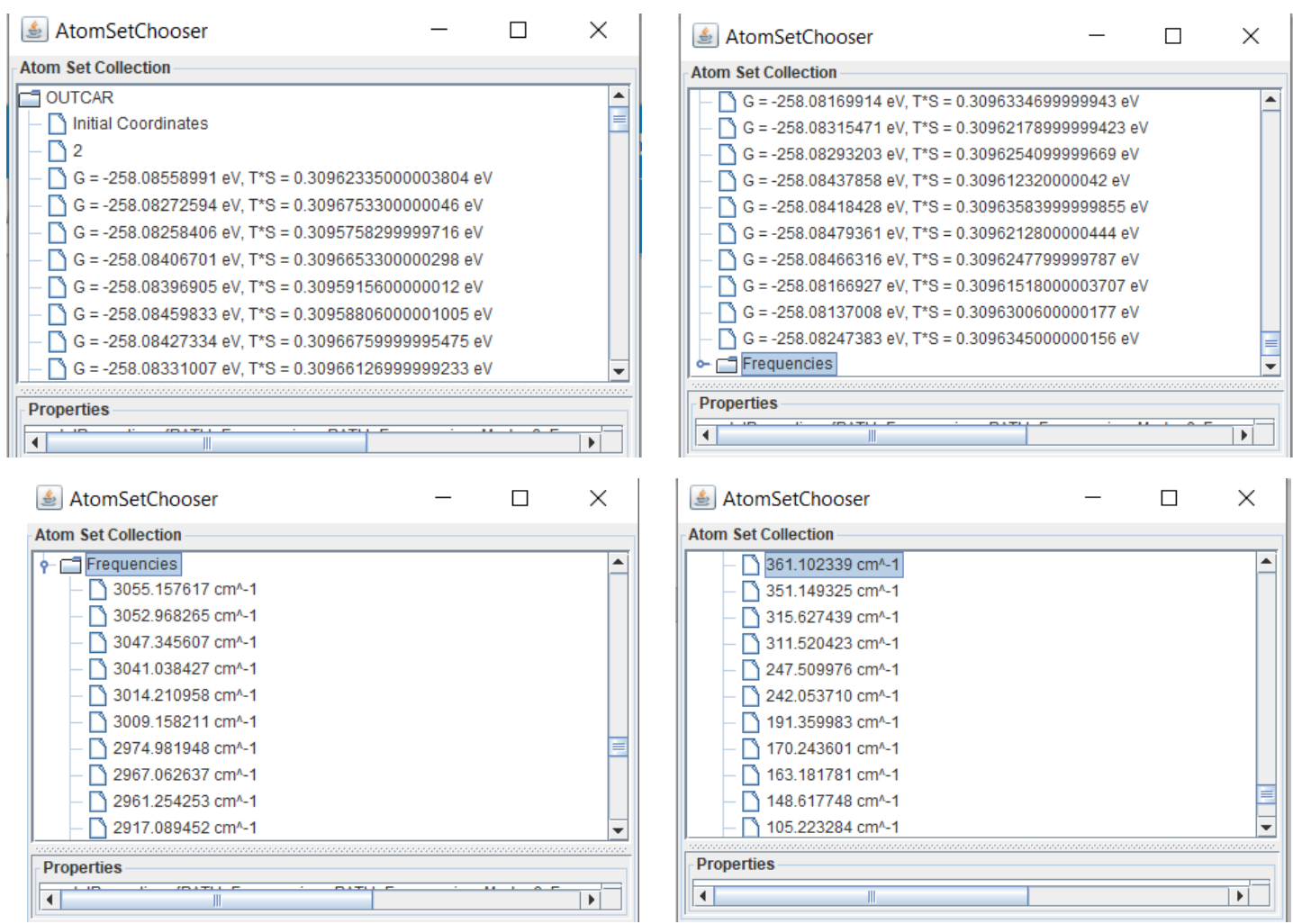

To visualize the vibration of each model, choose one frequency and then click the "vibration on" button (see below). (Here I choose the frequency $361.1 \mathrm{~cm}^{-1}$ as the example to illustrate the fitting procedures below)

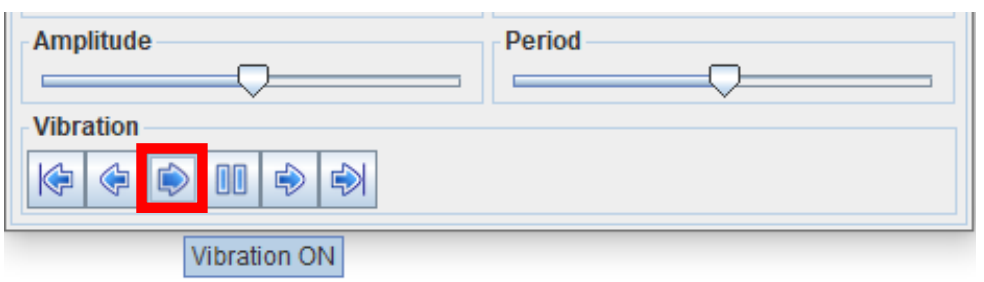

To make the vibration clearer, click "Display" button and select "Vector--> Scale 5", as well as "Vector--> 3 pixels":
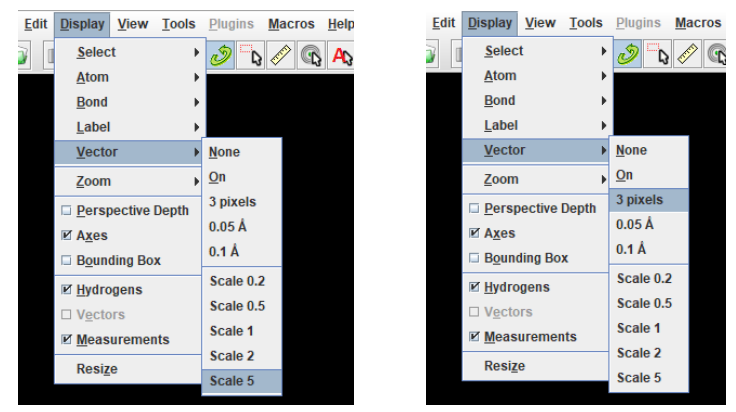

The vibrations from different views are listed below. Based on the the vibrations, the frequency $361.1 \mathrm{~cm}^{-1}$ corresponds to the angle $\mathrm{Au}(\mathrm{a})-\mathrm{S}(\mathrm{m})-\mathrm{C}$ bending mode. The value from DFT calculation 
is used as the reference value for the MD fitting. Note: the identification of vibrational mode in this step is very important since it determines whether you have the correct reference state, including vibrational frequency and vibrational mode, for the later MD fitting.
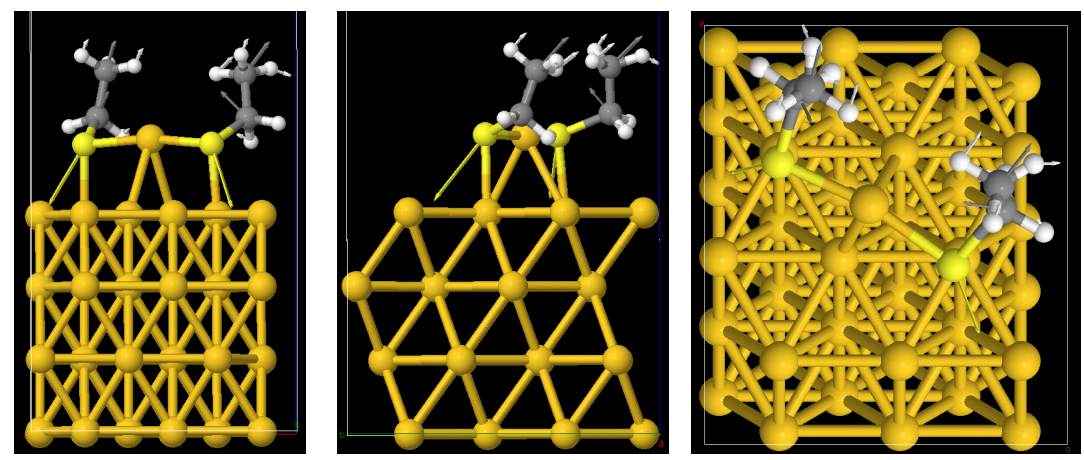

(3) We then need to optimize the structure with the minimize.exe executable: (a) double click the minimize.exe executable; (b) input the staple.xyz file and press the Enter button; (c) input the converged criterion (generally the default value is satisfactory) and the optimization will start after pressing press the Enter button; (d) the optimization would finish once it reaches the criteria. After the minimization, it would generate a new file named staple.xyz_2 and we need to replace the staple.xyz by the new staple.xyz_2. To maintain these two files, the old staple.xyz was named as staple_old.xyz
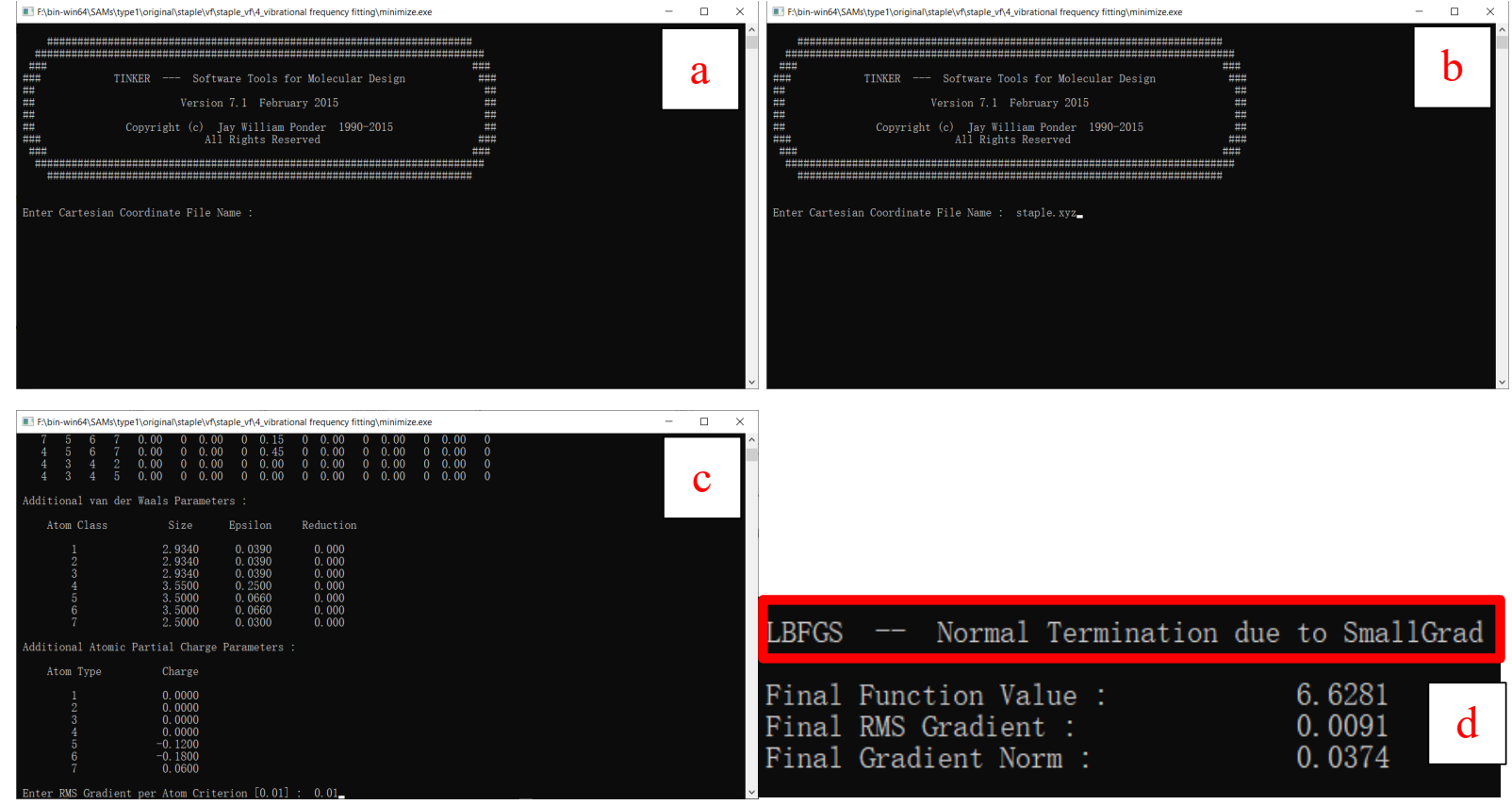

(4) Use the VIBRATE module to analyze the vibrational frequency:(a) double click the vibrate.exe executable; (b) input the staple.xyz file and press the Enter button. We can see the vibrational frequencies obtained from MD simulations. Basically, the number of vibrational frequencies from 
MD simulation should be the same as those from DFT calculation. In addition, each vibrational frequency corresponds to an integer number. (c) choose a vibrational frequency that is close to the DFT value (here we choose the $361.13 \mathrm{~cm}^{-1}$ since the DFT value that we selected is $361.10 \mathrm{~cm}^{-1}$ ) and then enter the corresponding value. After pressing the Enter button, it would generate a new file named staple.019. We then change it to be staple_19.xyz and copy staple.key to staple_19.key. (Note: You may find slight difference for the vibrational frequencies in (c), probably due to the fact that each optimization may not generate the structures with exactly same coordinates)
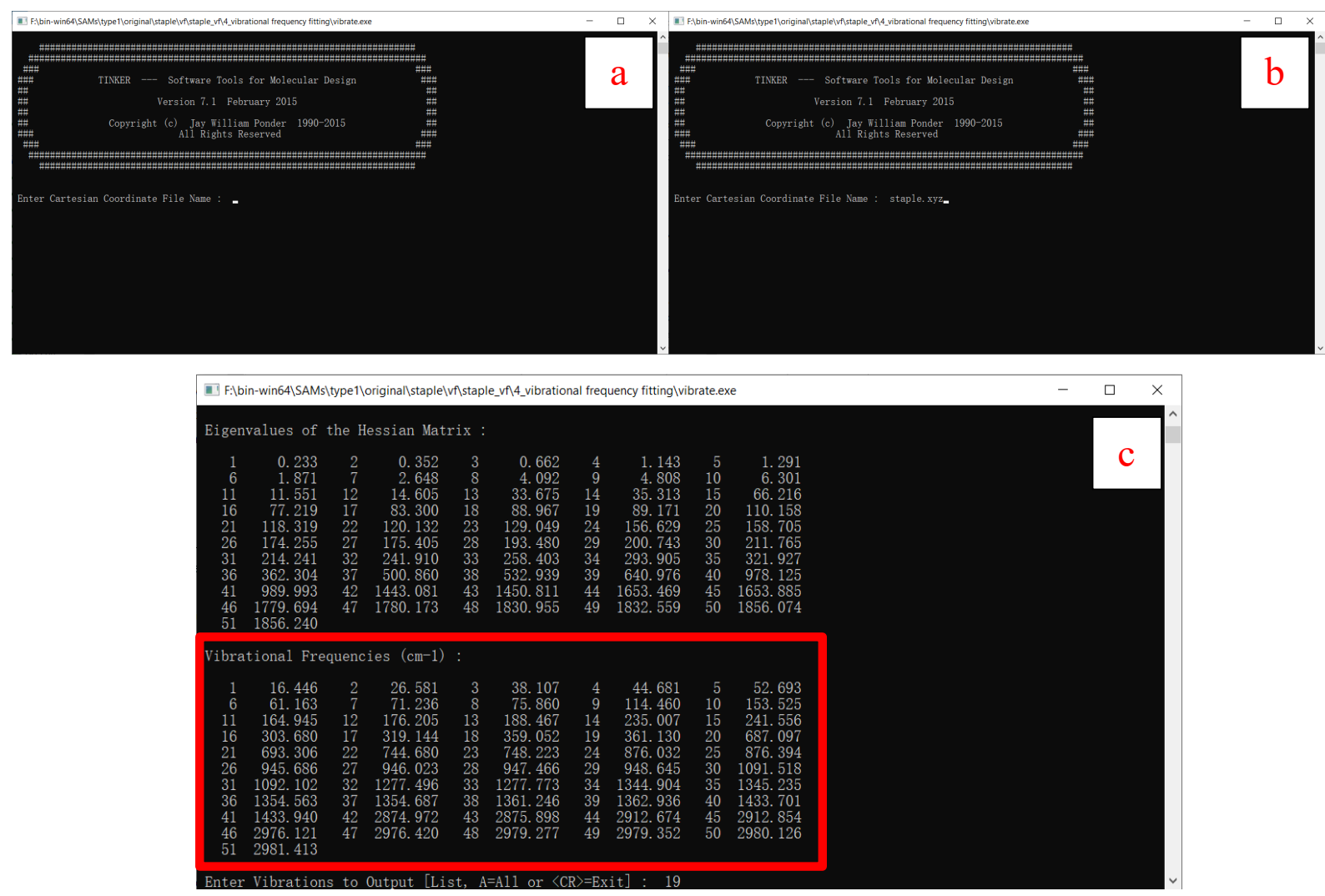

(5) Afterwards, we can use the Force Field Explorer to open the staple_19.xyz. Initially, you may observe only a part of the system (left figure of upper panel). To visualize the whole system, you need to do the following steps: (a) click and choose the staple_19.xyz on the left; (b) click "Display" and choose "Ball \& Stick". Then you can see the entire structure (right figure of lower panel). 

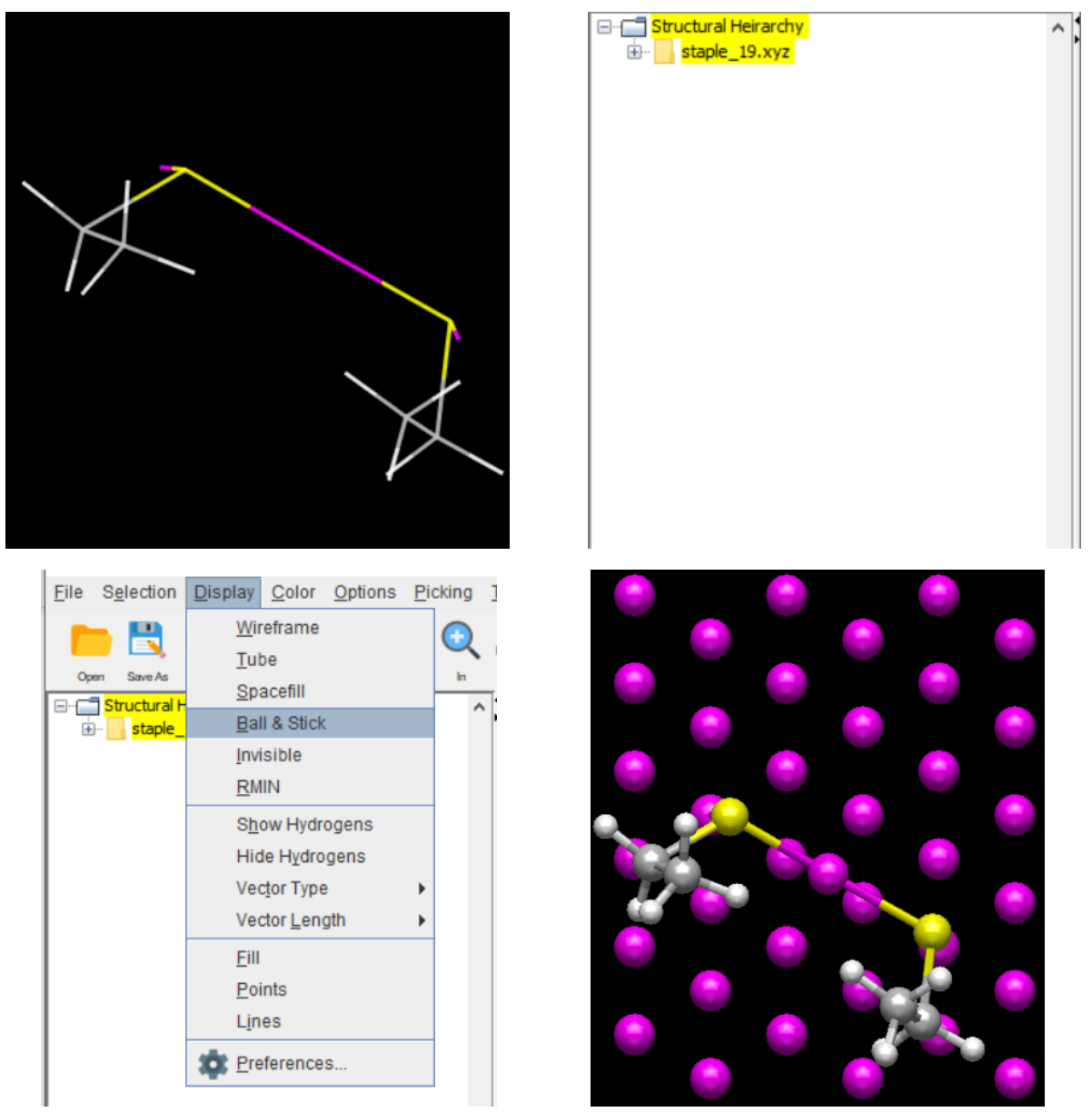

Indeed, the staple.xyz file contains 7 consecutive configurations and therefore we can use the Play function in Force Field Explorer to observe the vibration of system.

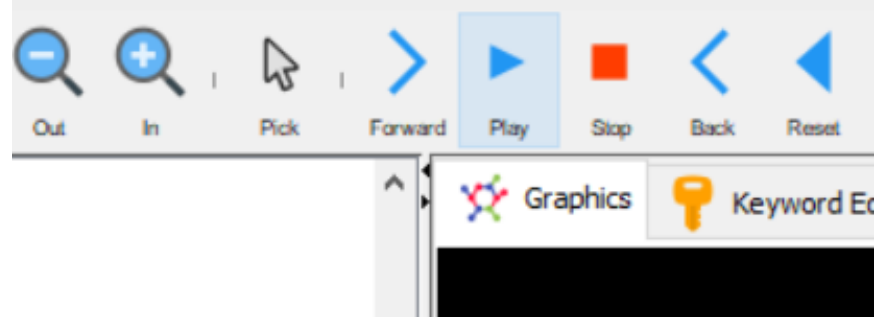

The aim of doing this is to compare the vibrational modes of the selected frequencies from both MD and DFT. If the vibrational modes of the two frequencies are the same, we conclude that the correct vibrational frequency in MD for the $\mathrm{Au}(\mathrm{a})-\mathrm{S}(\mathrm{m})-\mathrm{C}$ bending mode has been identified.

In our provided examples, the finally obtained values are considered as the initial guess values for the force constants and therefore we can easily find out the correct vibrational frequency and modes in MD simulation. In the initial stage, however, it is always difficult to choose an appropriate initial guess value. In this case, one of the valuable tips is that the initial guess values 
for the force constants of some new terms (bond, angle, and dihedrals) can be derived from the force field that contains similar local environment. With the initial guess values, we can do (3)-(5) of Step 4 to get the vibrational frequencies and modes for MD. Next, basically there would have two situations: (a) the obtained vibrational mode is the same to the one from DFT, but the vibrational frequency is different; (b) both the vibrational frequency and vibrational mode are distinct from the ones from DFT. For situation (a), once the vibrational mode is determined, you need to manually tune the force constant of corresponding term. Then we need to rerun (3)-(5) of Step 4 to get the vibrational frequency and compare it with the DFT reference value until they can agree with each other. For situation (b), we first need to run (4) of Step 4 to choose new vibrational frequencies and do the (5) of Step 4 to analyze the vibrational mode until we have the correct vibrational mode. After that, we only need to do the same operations as stated for situation (a) to get the force constant of targeted term. Use the aforementioned steps and operations for all interested bonds and angles, we can then obtain the desired force constants for them.

Part 2: Procedures to obtain the force constants for dihedrals via matching the torsional energy profiles. In this part, we use the $\mathrm{Au}(\mathrm{a})-\mathrm{S}(\mathrm{m})-\mathrm{C}-\mathrm{C}$ torsion term as the example to show how to fit the torsional energy profiles.

\section{Needed softwares:}

(1) Tinker-Ver. 7.1 (https://dasher.wustl.edu/tinker/)

(2) VASP-Ver. 5.4.1

(3) VESTA-Ver. 3.4.7 (http://jp-minerals.org/vesta/en/)

(4) Gauss View-Ver. 4.1

\section{Steps:}

1. Structural optimization (see the files in 1_optimization folder): this part of optimization is for the initial staple motif structure on the $\mathrm{Au}$ (111) surface, which is the same as step 1 of Part 1. 2. Convert CONTCAR to pdb (see the files in 2_contcar2pdb folder)

(1) Copy CONTCAR from 1_optimization folder to 2_contcar2pdb folder. Open "CONTCAR" in 2_contcar2pdb folder with VESTA 


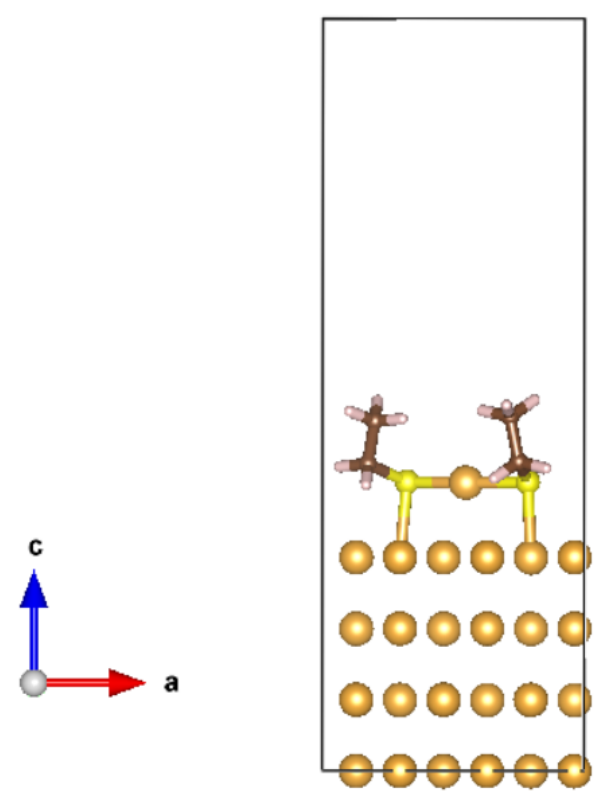

(2) From the "File" menu, choose "Export Data", save the file in pdb format in 2_contcar2pdb folder. By now, you shall have a file, named "staple.pdb".

(3) For the $\mathrm{Au}(\mathrm{a})-\mathrm{S}(\mathrm{m})-\mathrm{C}-\mathrm{C}$ torsion term, create 36 configurations with different dihedrals with an increase of $10^{\circ}$. A small interval like $5^{\circ}$ can be used around the equilibrium dihedral value.

(I) The following steps are used to create the bond connectivity between $\mathrm{S}$ atoms and $\mathrm{Au}$ atoms in the substrate in case there isn't any connectivity when you open the pdb file with GaussView: (a) Use GaussView to open the staple.pdb file; (b) Right click the mouse in main window and choose View-->Symbols to show the Symbols; (c) Right click the mouse in main window and choose View-->Builder; (d) choose the Modify Bond button; (e) select both S and Au atoms; (f) choose the second single bond and click OK button; (g) the bonds are created for S and Au atoms.
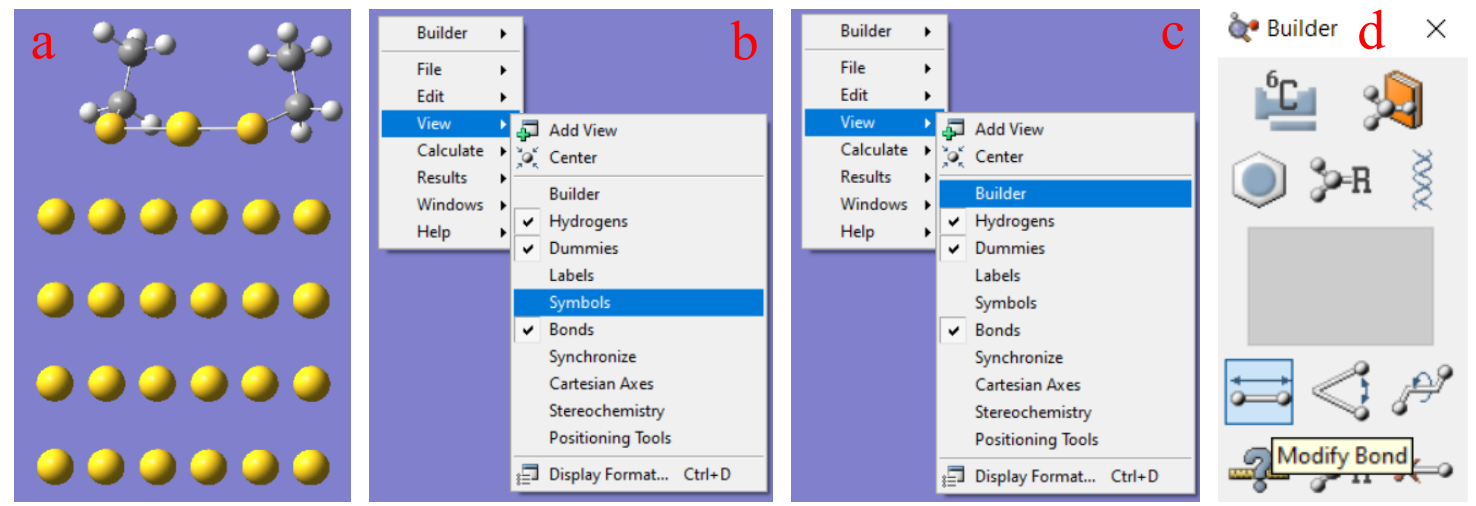

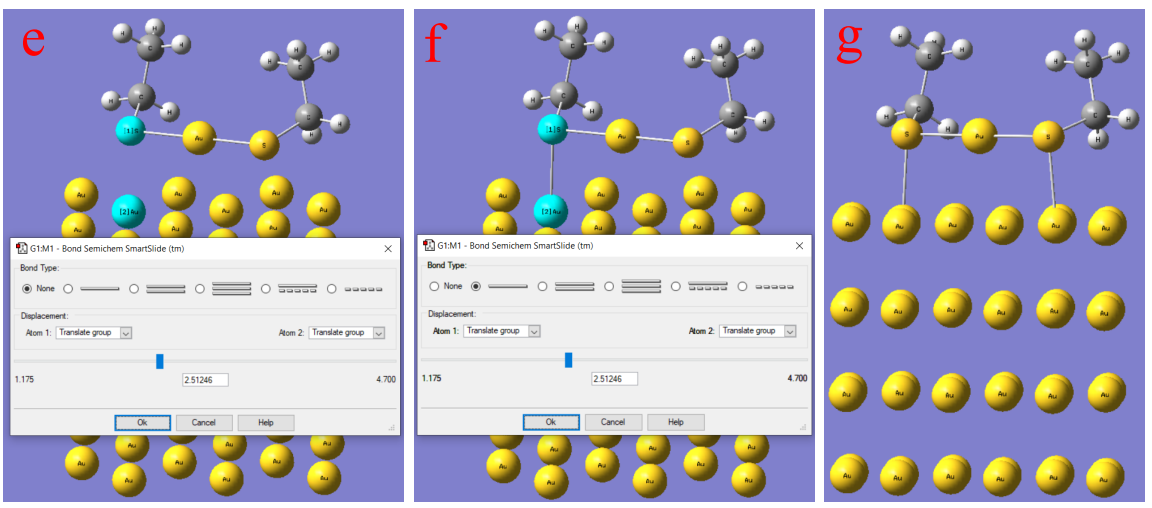

(II) The following steps are used to tune the dihedral for $\mathrm{Au}(\mathrm{a})-\mathrm{S}(\mathrm{m})-\mathrm{C}-\mathrm{C}$ torsional term based on the configuration of (I): (a) Right click the mouse in main window and choose View-->Builder; (b) choose the Modify Dihedral button; (c) select Au adatom, S, C, and C atoms in sequence; (d) for atom1, change the Rotate groups to Fixed; (e) change the dihedral to be $60.0^{\circ}$ and click OK. Here, we use the $60^{\circ}$ value as an example and the procedures are the same for the other dihedrals. One thing should be noted is that the dihedral in GaussView is from $-180^{\circ}$ to $180^{\circ}$ and therefore the dihedral in the range of $-180^{\circ}$ to $0^{\circ}$ corresponds to $180^{\circ}$ to $360^{\circ}$. (f) From the "File" menu, choose "Save...", save the file in pdb format, named as staple_60.pdb in the 3_pdb2poscar folder.
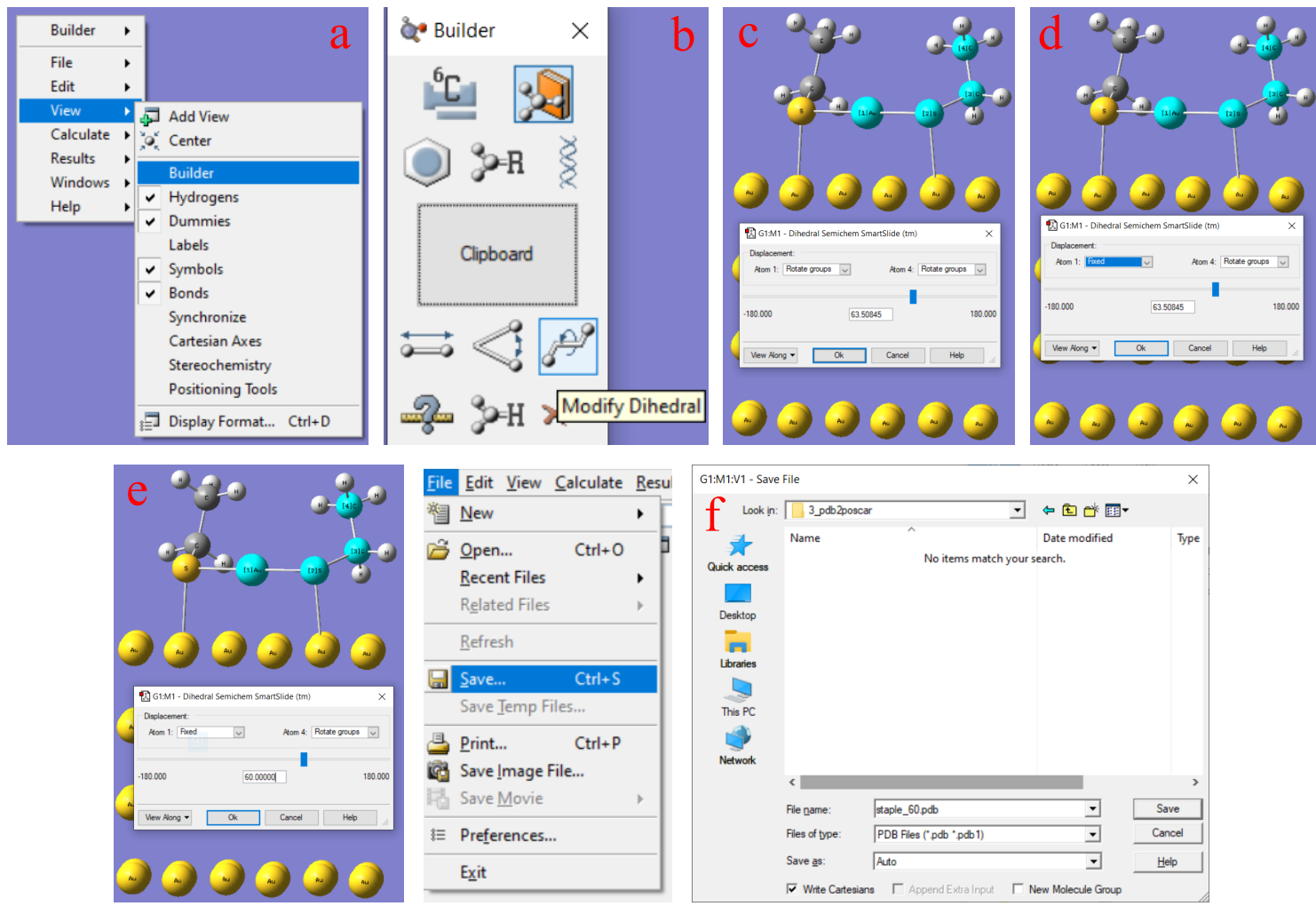

(III) Use the pdb2pos.f90 code to convert the staple_60.pdb file to POSCAR (the files are in the 3_pdb2poscar folder). Open the POSCAR with VESTA to check the structure. 


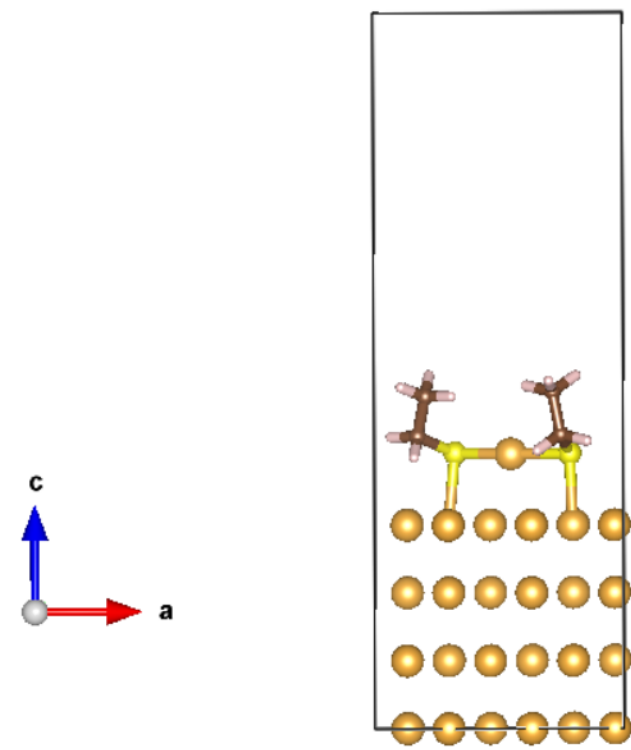

(IV) Structural optimization with VASP (see files in 4_optimization for dihedral angle folder): this part of optimization is for the $\mathrm{Au}(\mathrm{s})-\mathrm{S}(\mathrm{m})-\mathrm{C}-\mathrm{C}$ torsional term with specific dihedral. (a) copy the POSCAR from 3_pdb2poscar folder to 4_optimization for dihedral angle folder; (b) prepare additional three files, namely, INCAR, POTCAR, and KPOINT. After finishing the optimization, it would generate the CONTCAR file (see the files in 4_optimization for dihedral angle folder) (V) Convert the CONTCAR file to .xyz file. In fact, we can use the steps in Part 1 to get the .xyz file from CONTCAR file. However, during the fitting process for the torsional terms, there are hundreds of CONTCAR to be converted into .xyz file. Therefore, a Fortran code cont2xyz.f90 has been prepared to simplify the conversion steps (see the files in 5_contcar2xyz folder).

(VI) Copy the final staple.key file from Part 1 and staple_60.xyz from 5_contcar2xyz folder to the 6_energy analysis folder and change the staple.key to be staple_60.key. The following steps are carried out to calculate the total energy for MD: (a) double click the analyze.exe executable; (b) input the staple_60.xyz and press the Enter button; (c) choose the third one and input the letter E; (d) we can get the MD total energy for the $\mathrm{Au}(\mathrm{a})-\mathrm{S}(\mathrm{m})-\mathrm{C}-\mathrm{C}$ term with a dihedral of $60^{\circ}$.
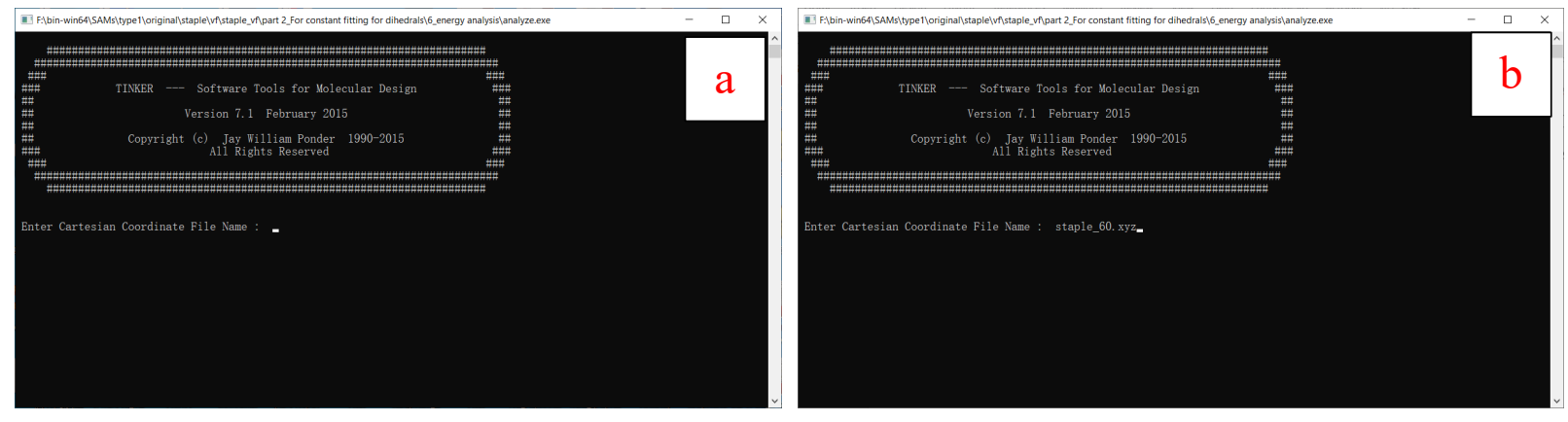

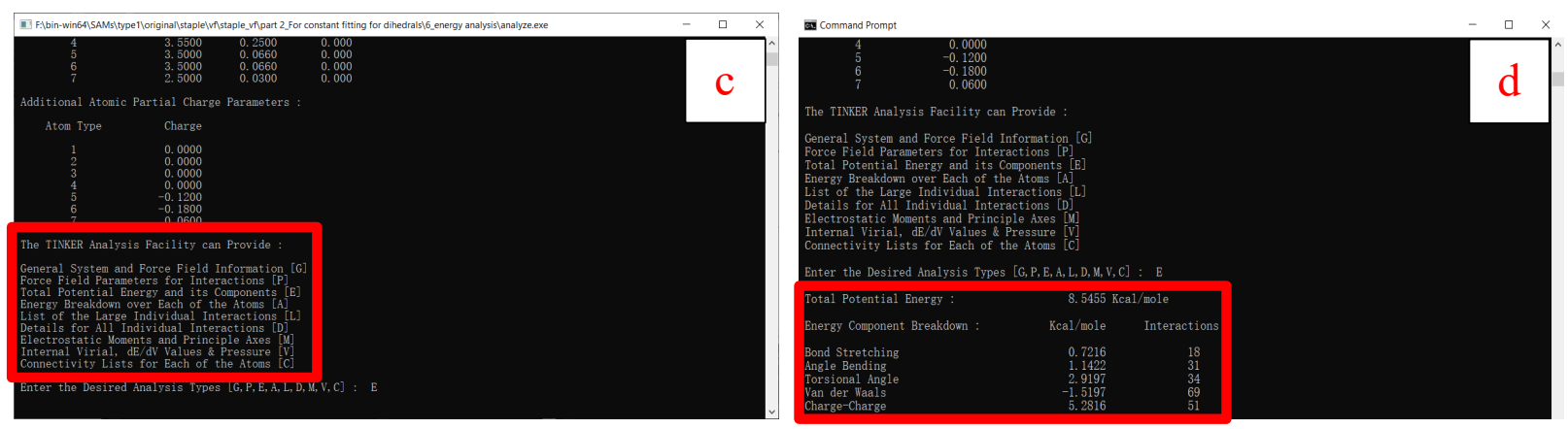

(VII) Use the same method, we can get the MD total energy for the $\mathrm{Au}(\mathrm{a})-\mathrm{S}(\mathrm{m})-\mathrm{C}-\mathrm{C}$ term with other dihedrals. Finally, we can obtain the MD total energy in the range of $-180^{\circ}$ to $180^{\circ}$. For both DFT and MD energies, find out the configuration with the lowest energy, which is then used as the reference value to calculate the energy difference as we display in the manuscript.

(VIII) Plot and compare the energy profiles from both DFT and MD. If there is a good agreement between them, the force constant would be the one that we want for the Au(a)-S(m)-C-C term. If not, we need to tune the force constant in the .key file and rerun the energy analysis as we described in VI) and VII) until we can get a good match between the DFT and MD results.

Part 3: The computational resources

In part 1 of the Supporting Information 2, the CPU time for the DFT-based vibrational frequency calculation is about 4.6 and 1.6 hours for staple motif and bridge models, respectively, by using 48 cores. For the vibrational frequency fitting from MD, the time depends on the initial guess values of the force constant and it may vary from several days to one week. In part 2 of the Supporting Information 2, the computational resources mainly focus on the DFT optimization for the structures with different dihedrals and the torsional energy profiles fitting from MD. In regard to the optimization of each dihedral, the CPU time for the staple motif model varies from 0.2 (the most stable structure) to 12.5 hours (the least stable structure) by using 48 cores, and the optimization jobs are more than 150. In the case of bridge model, the corresponding CPU time varies from 0.5 (the most stable structure) to 3.7 hours (the least stable structure) with the same number of cores, and the optimization jobs are more than 70 . For the force field validation simulations, the CPU time is about 40 hours for AIMD simulations to run 10 ps with 48 cores, while the corresponding time is about 100 hours for MD simulations to run $10 \mathrm{~ns}$ with 24 cores. All the calculations mentioned above were performed by using the high-performance supercomputers at University of Oklahoma and Nanjing Tech University. 